\title{
Repeatability of glucocorticoid hormones in vertebrates: A meta-analysis
}

\author{
Kelsey L Schoenemann ${ }^{\text {Corresp.. }}{ }^{1}$, Frances Bonier ${ }^{1}$ \\ ${ }^{1}$ Biology Department, Queen's University, Kingston, Ontario, Canada \\ Corresponding Author: Kelsey L Schoenemann \\ Email address: kelsey.schoene@gmail.com
}

We often expect that investigations of the patterns, causes, and consequences of amongindividual variation in a trait of interest will reveal how selective pressures or ecological conditions influence that trait. However, many endocrine traits, such as concentrations of glucocorticoid (GC) hormones, exhibit adaptive plasticity and, therefore, do not necessarily respond to these pressures as predicted by among-individual phenotypic correlations. To improve our interpretations of among-individual variation in GC concentrations, we need more information about the repeatability of these traits within individuals. Many studies have already estimated the repeatability of baseline, stress-induced, and integrated GC measures, which provides an opportunity to use meta-analytic techniques to investigate 1) whether GC titers are generally repeatable across taxa, and 2) which biological or methodological factors may impact these estimates. From an intensive search of the literature, we collected 91 GC repeatability estimates from 47 studies. Overall, we found evidence that GC levels are repeatable, with mean repeatability estimates across studies ranging from 0.230 for baseline levels to 0.386 for stress-induced levels. We also noted several factors that predicted the magnitude of these estimates, including taxon, sampling season, and lab technique. Amphibians had significantly higher repeatability in baseline and stress-induced GCs than birds, mammals, reptiles, or bony fish. The repeatability of stress-induced GCs was higher when measured within, rather than across, life history stages. Finally, estimates of repeatability in stress-induced and integrated GC measures tended to be lower when GC concentrations were quantified using commercial kit assays rather than in-house assays. The extent to which among-individual variation in GCs may explain variation in organismal performance or fitness (and thereby inform our understanding of the ecological and evolutionary processes driving that variation) depends on whether measures of GC titers accurately reflect how individuals differ overall. Our findings suggest that while GC titers can reflect some degree of consistent differences among individuals, they frequently may not. We discuss how our findings contribute to interpretations of variation in GCs, and suggest routes for the design and analysis of future 
research. 
Title: Repeatability of glucocorticoid hormones in vertebrates: a meta-analysis

Authors: Kelsey L. Schoenemann ${ }^{\mathrm{a}}$, Frances Bonier ${ }^{\mathrm{a}}$

Affiliations: ${ }^{a}$ Department of Biology, Queen's University, Kingston, Ontario, Canada K7L 3N6

Corresponding author: Kelsey Schoenemann; kelsey.schoene@gmail.com

\begin{abstract}
We often expect that investigations of the patterns, causes, and consequences of amongindividual variation in a trait of interest will reveal how selective pressures or ecological conditions influence that trait. However, many endocrine traits, such as concentrations of glucocorticoid (GC) hormones, exhibit adaptive plasticity and, therefore, do not necessarily respond to these pressures as predicted by among-individual phenotypic correlations. To improve our interpretations of among-individual variation in GC concentrations, we need more information about the repeatability of these traits within individuals. Many studies have already estimated the repeatability of baseline, stress-induced, and integrated GC measures, which provides an opportunity to use meta-analytic techniques to investigate 1) whether GC titers are generally repeatable across taxa, and 2) which biological or methodological factors may impact these estimates. From an intensive search of the literature, we collected 91 GC repeatability estimates from 47 studies. Overall, we found evidence that GC levels are repeatable, with mean repeatability estimates across studies ranging from 0.230 for baseline levels to 0.386 for stressinduced levels. We also noted several factors that predicted the magnitude of these estimates, including taxon, sampling season, and lab technique. Amphibians had significantly higher repeatability in baseline and stress-induced GCs than birds, mammals, reptiles, or bony fish. The repeatability of stress-induced GCs was higher when measured within, rather than across, life history stages. Finally, estimates of repeatability in stress-induced and integrated GC measures tended to be lower when GC concentrations were quantified using commercial kit assays rather than in-house assays. The extent to which among-individual variation in GCs may explain variation in organismal performance or fitness (and thereby inform our understanding of the ecological and evolutionary processes driving that variation) depends on whether measures of GC titers accurately reflect how individuals differ overall. Our findings suggest that while GC titers can reflect some degree of consistent differences among individuals, they frequently may not. We discuss how our findings contribute to interpretations of variation in GCs, and suggest routes for the design and analysis of future research.
\end{abstract}




\section{Introduction}

Since the development of immunoassays that allow the measurement of hormones in relatively small-volume tissue samples (Ekins, 1960; Yalow \& Berson, 1960), the number of studies investigating the patterns, causes, and consequences of endocrine trait variation has soared. Early work in this field described variation in hormone concentrations across species, populations, and life history stages (e.g., Boswell et al., 1994; Klosterman et al., 1986; Pancak and Taylor, 1983), while more recent work often measures among-individual variation in multiple endocrine traits, including hormone concentration, receptor density, binding protein concentration, and endocrine axis responsiveness (e.g., Breuner et al., 2006; Bizon et al., 2001; Lattin \& Romero, 2014; Liebl, Shimizu \& Martin, 2013). Thus, much of our understanding of how selection has shaped these traits derives from comparative studies that determine how conserved or variable hormones, receptors, or their effects are across taxa, or how those traits vary among individuals with geography, phylogeny, or other traits of interest (e.g., Bókony et al., 2009; Eikenaar et al., 2014; Heidinger et al., 2006). Yet, traits that exhibit adaptive plasticity, such as hormone titers, might not respond to selective pressures or ecological conditions as predicted by among-individual phenotype-fitness correlations (Stinchcombe et al., 2002; Bonier et al., 2009; Bonier \& Martin, 2016). Moving beyond this comparative approach to better understand endocrine trait evolution requires knowledge about heritable individual differences in evolutionarily-important traits because natural selection acts upon this heritable variation at the individual level (Bennett, 1987; Williams, 2008). However, the extent to which variation in hormone levels can be attributed to fixed individual differences is poorly understood. 
52 environmental changes, and referred to as flexibility in some contexts (sensu Bonier and Martin,

53 2016). The plasticity of GC titers helps organisms achieve allostasis (from the Greek allo

54 meaning 'variable'+ stasis meaning 'stand'; thus, 'stability through change'). Allostasis can refer

55 to 1) the process that maintains or reestablishes the physiological parameters essential for life,

56 such as $\mathrm{pH}$, oxygen levels, or blood pressure (i.e., homeostasis), even while regulatory thresholds

57 may change with environmental conditions, as well as 2) the organism's ability to produce the

58 mediators, such as hormones, immune-signaling proteins, and (para)sympathetic activity, that

59 promote physiological adaptation to new conditions (Romero, Dickens \& Cyr, 2009; McEwen \&

60 Wingfield, 2007). In the case of GC hormones, rapidly elevating circulating concentrations (i.e.,

61 via activation of the hypothalamic-pituitary-adrenal [HPA] axis) promote behavioral and

62 physiological changes that enable an organism to respond to and recover from acute challenges,

63 and the modulation of baseline HPA activity supports responses to predictable changes in

64 energetic demands across daily or seasonal cycles (Sapolsky, Romero \& Munck, 2000; Romero,

65 2004; Wingfield, 2005; Romero, Dickens \& Cyr, 2009). Failure to acknowledge, measure, or

66 control for these sources of within-individual variation can diminish our ability to detect

67 biologically significant patterns in GC secretion among individuals.

Estimating the repeatability (i.e., consistency over time or across contexts) of GC titers is one technique for assessing and potentially avoiding this pitfall. Multiple test statistics have been used to estimate the repeatability of a trait in a population (e.g., Spearman rank and Pearson correlation coefficients), but the intraclass correlation coefficient (ICC) is the most prevalent in recent literature (Sokal and Rohlf 1995; Nakagawa and Schielzeth, 2010). The repeatability of GCs within individuals can be used to determine the degree to which inferences made about GC measures may be generalized beyond providing information about the individuals at the time of 
75 sampling (e.g., Bosson et al., 2009; Harris et al., 2016; Wada et al., 2008). Moreover,

76 repeatability itself may reflect the ability or strategy of an individual to cope with a challenge

77 and, thus, is worthy of study in its own right (Careau, Buttemer \& Buchanan, 2014; Roche,

78 Careau \& Binning, 2016). Finally, estimates of repeatability can approximate the upper limit of

79 heritability of individual variation and, thereby, the extent to which natural selection can shape a 80 trait (Falconer and Mackay 1996; but see Dohm, 2002). Perhaps in recognition of these points,

81 many studies have estimated the repeatability of GC measures (e.g., Cook et al., 2012; Narayan

82 et al., 2013a; Romero and Reed, 2008; Wada et al., 2008). The availability of these estimates

83 provides an opportunity to investigate whether GCs are generally repeatable across taxa, and

84 how biological or methodological factors may impact these estimates.

85 To date, researchers have estimated the repeatability of GC levels in every class of

86 vertebrates, and across various environmental contexts and spans of time. A meta-analysis of

87 repeatability estimates across these studies could determine whether GCs are generally

88 repeatable, and whether variation in the magnitude of repeatability can be explained by

89 biological or methodological factors. For example, meta-analyses of behavior and metabolic rate

90 repeatabilities have provided evidence of significant trait repeatability, as well as differences in

91 repeatability according to sex, sampling interval, captive condition, and taxon (Nespolo \&

92 Franco, 2007; Bell, Hankison \& Laskowski, 2009; White, Schimpf \& Cassey, 2013). Here, we

93 similarly seek to investigate sources of variation in estimates of repeatability of GCs.

94 Specifically, the aim of this meta-analysis is to: 1) summarize the available evidence of

95 repeatability of GC concentrations; and 2) identify biological and methodological factors that

96 predict variation in the magnitude of GC repeatability. 
98

99

100

101

102

103

104

105

106

107

108

109

110

111

112

113

114

115

116

117

118

119

120

\section{Methods}

\subsection{Literature Search}

We performed literature searches on Google Scholar between March 2016 and November 2017 using the terms: "repeatab*," "consisten*," "glucocorticoid," "cortisol”, "corticoster*”, “repeated measure," and "individual variation." We identified 716 records in these searches. We screened the titles and abstracts of these records, looking for papers that estimated the repeatability (or 'consistency' or 'individuality') of concentrations of glucocorticoid hormones in a variety of tissues (e.g., blood, saliva, feces, feathers). To be selected for inclusion in this analysis, a study needed to have assessed repeated measurements from the same individual and estimated a repeatability coefficient (e.g., Spearman rank, Pearson, or ICC). We excluded duplicate and irrelevant articles and those that did not meet our inclusion criteria (Fig. 1). We also checked reference lists of selected papers to find additional studies that were not identified in the initial search. Lastly, we included 3 studies that collected repeated measurements of hormone concentrations from the same individuals but did not estimate repeatability, when we could obtain the original data to calculate repeatability.

\subsection{Repeatability Estimates}

We extracted repeatability estimates from the selected studies and categorized them as representing either initial, response, or integrated GC repeatability measures. We used the category initial to group repeatability estimates of GC titers measured in circulation within a time period expected not to reflect the acute stress of capture, response for repeatability estimates of the elevated GC titers following an acute capture, handling, or confinement stress, and integrated for repeatability estimates of GC titers that represent hormone secretion over a 
121 relatively long period of time (e.g., GC concentrations in feces, feathers, and saliva). If the study

122 did not calculate repeatability, then, where possible, we obtained the original data and calculated

123 an ICC repeatability, using the 'rptR' package (version: 0.9.2) in R (version 3.4.0, 2017-04-21)

124 (Nakagawa \& Schielzeth, 2010).

125

\subsection{Statistical Analysis}

127

We harvested information about several methodological and biological factors associated

with each repeatability estimate and categorized these data for analysis (Table 1). We used linear mixed-effect models (LMMs) with the 'Ime4' package (version: 1.1.13) to investigate variation in repeatability estimates. We included study identity as a random effect to control for potential bias arising from non-independence of multiple estimates derived from the same study

132 (Nakagawa \& Santos, 2012). One study, however, was coded with two independent study

133 identities because the datasets included in this one study were collected by two different

134 researchers, on different species, in different field sites (Ouyang, Hau \& Bonier, 2011). We

135 constructed separate LMMs to address each of the following questions with initial, response, or

136 integrated $\mathrm{GC}$ repeatability measures:

137 1. Does sampling regime predict repeatability? To answer this question, we evaluated the

138 following fixed effects: sample size, average time span between samples, and average 139 number of samples.

140 2. Does subject biology or sampling environment predict repeatability? We evaluated the fixed

141 effects taxonomic class, sex, whether samples were collected within or across life history

142 stage, captive condition, and experimental manipulation (whether or not some/all individuals

143 underwent a stressful manipulation intended to produce a response [not including routine 
144 capture and handling stress] at some point during the course of the study). We lacked

145 sufficient power to evaluate the effect of age because we identified only two estimates of

146 repeatability that were measured solely in juveniles or immature individuals. We also

147 evaluated the fixed effect of life history stage (breeding, non-breeding, or pre-breeding) in a

148 subset of GC repeatability estimates measured within a single stage.

149 3. Do laboratory or statistical techniques predict repeatability? We evaluated the fixed effects

150 use of an in-house assay or commercial assay kit, use of a radioactive or enzymatic tracer,

151 and whether or not the statistical analysis incorporated confounding factors (i.e., if the

152 repeatability estimate controlled for correlations between GCs and factors such as the time or

153 year of sampling, and the breeding status, age, or body mass of the individuals sampled).

With the exception of models that included sample size as a fixed factor (question 1,

156 above), we weighted each estimate by its sample size to account for differences in statistical

157 power among studies. Thus, estimates from larger studies had a greater influence in the models.

158 We verified the normality of model residuals with a Shapiro test. When model residuals failed to

159 meet the assumption of normality, we square-root transformed the data. To identify important

160 predictors of repeatability, we coded global models with all candidate variables included as main

161 effects and used the dredge function from the 'MuMIn' package (version: 1.15.6) to rank

162 recombinant models with the Akaike's information criterion corrected for small sample sizes

163 (AICc). We did not include any interaction terms in our models, due to small sample sizes. We

164 report effect size and p-values from either the best-fit model or, when more than one model was

165 ranked within $2 \triangle \mathrm{AICc}$ of the best-fit model, from a conditional average of all top models. Due

166 to the small sample size of integrated measures available to address question 2, we compared the 
167 saturated model to a null model using an F-test with Kenward-Roger approximation using the

168 'pbkrtest' package (version: 0.4-7) (Kenward \& Roger, 1997; Halekoh \& Højsgaard, 2014). For

169 some non-ordinal variables (e.g., taxonomic class, sampling interval), it is more informative to

170 consider the significance of the factor as a whole rather than at specific levels; therefore, in such

171 cases, we performed a Type III ANOVA with Satterthwaite approximation for degrees of

172 freedom using the 'ImerTest' package (version: 2.0-33) to obtain p-values (Kuznetsova,

173 Brockhoff \& Christensen, 2016).

174 In addition to including study identity as a random effect, we employed several other

175 methods to address potential bias or pseudo-replication. First, we did not include redundant

176 estimates from the same study nor re-analyses of the same data. Second, we assessed the

177 independence of multiple repeatability estimates originating from the same study. If a single GC

178 measure is correlated among multiple groups of individuals (e.g., similarly low initial GC

179 repeatability in males and females from same population), then we might expect multiple

180 repeatability estimates of the same population to be non-independent. To test for this effect, we

181 performed a linear regression analysis with those studies that reported more than one estimate to

182 test whether the number of estimates of repeatability in a study was associated with repeatability

183 (Nespolo \& Franco, 2007; Bell, Hankison \& Laskowski, 2009). We did not find a relationship

184 between initial repeatability and the number of estimates reported in the study (linear model:

185 initial $n=37, p=0.127$ ), and no studies of integrated repeatability reported more than two

186 estimates. We did find a significant negative relationship between the number of estimates of the

187 repeatability of response GCs and their magnitude ( $n=31, \beta=-0.10, p=0.002)$, however, this

188 relationship was driven by a single study that reported multiple estimates of 0.00 repeatability. 
189 Thus, our inclusion of study identity as a random effect in all models was deemed sufficient to

190 control for non-independence of multiple estimates from the same study.

191 Finally, to determine whether GCs are generally repeatable across all studies, we first

192 needed to assess whether the estimates we obtained from the literature represent a random

193 sample of the 'true' repeatability of GC titers. Given that the primary focus of most studies

194 included in this analysis was not to estimate repeatability, we expect publication bias is unlikely

195 to be an important source of bias for our results. Nevertheless, we assessed this and other

196 potential biases directly by plotting every estimate against its sample size in funnel plots. Upon

197 finding these plots symmetrical (Supplemental Fig. 1), we concluded that bias is unlikely (Egger

198 et al., 1997). Therefore, we calculated 95\% confidence intervals around the mean repeatabilities

199 of initial, response, and integrated measures across all studies, regardless of taxon, using 1000

200 bootstrap samples of the data with replacement. We interpret a confidence interval that does not

201 overlap zero as indicating that the mean GC repeatability estimate is greater than zero (i.e., the

202 GC measure is, on average, somewhat repeatable), and interpret confidence intervals that do not

203 overlap each other as indicating different repeatabilities.

204

205 3. Results

206

\subsection{Summary of the data set}

We identified 47 studies that met our criteria for inclusion, from which we extracted 91

estimates of GC repeatability (summarized in Table 2, see Supplementary Information for complete dataset). In brief, more estimates were made of initial or response measures than of

211 than two-thirds of the estimates originated from studies of birds. Free-ranging populations of 
212 adults with both sexes combined were more often studied than captive populations, juveniles or

213 immatures, or separately for the sexes. About three-quarters of the estimates spanned a sampling

214 interval of less than one year. The majority of estimates came from repeated measurement within

215 the same life history stage and, of those measured within a stage, more were derived from

216 measurements taken during the breeding season. Finally, the ICC was the most common

217 repeatability estimate reported, with 42 studies reporting an ICC and only 4 reporting either

218 Pearson or Spearman correlations; in one study, the authors did not clearly report method used

219 nor respond to our requests for information.

220

221

222

\subsection{Repeatability of GCs}

Overall, GC levels were moderately repeatable, with mean repeatabilities ranging from

224 for response measures (Fig. 2). Moreover, the 95\% confidence intervals around the mean

225 repeatability of all three types of measures did not overlap zero (initial: 0.230 [0.162, 0.294],

226 response: $0.386[0.318,0.449]$, integrated: $0.320[0.235,0.410])$. As indicated by non-

227 overlapping confidence intervals, the mean repeatability of response measures is greater than 228 that of initial measures.

\subsection{Relationships between repeatability and biological or methodological factors}

231 3.3.1 Does sampling regime predict repeatability?

232 We found little evidence that sample size, time span between samples, or number of

233 samples predicts GC repeatability. The null was the best-fit model for integrated measures and,

234 while number of measurements and sample size were retained in top models of initial and 
235 response measures (Supplementary Table 1), we did not find evidence that initial or response

236 repeatability varied significantly with these factors (model average: all $p>0.12$ ). Sampling

237 interval, however, was retained in top models of response measures and, on average,

238 repeatability was greater when repeated measurements were collected within 8-14 days of each

239 other $(0.607, n=8)$, compared to either shorter ( $0-7$ days; $0.327, n=5)$ or longer $(15-365+$ days;

$2400.324, n=24$ ) intervals (Type III ANOVA; $n=37, F(5,35)=2.840, p=0.030$ ).

241

242 3.3.2 Does subject biology or sampling environment predict repeatability?

243 Taxonomic class was retained in the top models explaining variation in repeatability

244 estimates for both initial and response measures (Supplemental Table 2). On average,

245 amphibians had higher initial and response repeatability $(0.833, n=4 ; 0.786, n=4$, respectively)

246 than birds $(0.162, n=35 ; 0.318, n=21$ ), mammals ([no initial $\mathrm{GC}$ repeatability estimates in

247 mammals]; 0.446, $n=5)$, reptiles $(0.270, n=1 ; 0.21, n=2)$, or fish $(0.201, n=2 ; 0.359, n=5)$

248 (Fig. 3; Type III ANOVA; initial: $n=38, F(3,38)=9.359, p<0.0001$; response: $n=27$,

$249 F(4,23)=4.984, p=0.005)$. While sex was retained in the top models of initial measures, we did

250 not find strong evidence that repeatabilities varied by sex (model average: all $p>0.15$ ).

251 Estimates of response repeatability were higher when derived from measurements within a life

252 history stage $(0.502, n=22)$ than when derived from measurements across stages $(0.072, n=5)$

253 (Supplemental Table 3; model average: $n=27, \beta=0.235, p=0.007$ ). Neither experimental

254 manipulation nor captive condition was retained in any top models. The global model evaluating

255 integrated measures was not better-fit than the null (F-test: $n=10, F(7,3023)=0.191$,

$256 p=0.988)$. 
Finally, in the subset analyses of repeatability estimates measured within a life history

258 stage, we found little evidence that life history stage (breeding, non-breeding, or pre-breeding)

259 predicts repeatability. The null model was the best-fit model for initial and response measures

260 (Supplemental Table 2). However, a univariate model including life history stage performed

261 better than the null for integrated measures, where repeatability was on average higher in the

262 non-breeding season $(0.555, n=3)$ compared to breeding $(0.266, n=5$; F-test: $n=8, F(1,2370)$

$263=10.7, p=0.001)$.

264

265

266

267

268

269

270

271

272

273

274

275

276

277

278

279

\subsubsection{Do laboratory or statistical techniques predict repeatability?}

Assay type (in-house or kit) was retained in top models of initial, response, and

integrated measures, while assay tracer was retained in the top models of initial and integrated measures (Supplemental Table 4). Repeatabilities of initial and integrated hormone

concentrations measured with RIA were lower than those measured with EIA, although this difference was not as evident for initial measures (Supplemental Table 5; model average initial: $n=40, \beta=-0.132, p=0.071$; integrated: $n=11, \beta=-0.194, p=0.024)$. In addition, the repeatabilities of response measures were lower when measured with a kit than those measured with an in-house assay, and tended to be lower for repeatability of integrated measures

(Supplemental Table 5; model average: response: $n=35, \beta=-0.184, p=0.040$; integrated: $n=11, \beta=-0.172, p=0.062$ ). Finally, whether or not confounding factors were controlled was retained in one top model of response measures, however, we did not find strong evidence that repeatability varied with this factor (Supplemental Table 5; model average: $n=35, \beta=0.101$, $p=0.340)$ 


\section{Discussion}

281 To better understand individual variation in GCs, we summarized published estimates of

282 GC repeatability and identified factors that predicted the magnitude of those estimates. We found

283 measures of initial, response, and integrated GCs had mean repeatabilities of $0.230,0.386$, and

2840.320 , respectively, with response repeatability estimates greater than initial repeatability. In

285 general, this finding suggests that measures of GC titers reflect a moderate degree of consistent

286 differences among individuals, however, some measures were more or less repeatable, depending

287 on how the biological sample was collected and analyzed or which individuals were sampled.

288 Specifically, we found that some estimates of GC repeatability were greater in amphibians, when

289 all samples from an individual were collected within a single life history stage, and when

290 samples collected within a life history stage came from the non-breeding season. We also found

291 some evidence that GC repeatability was greater when hormone concentrations were measured

292 using an in-house immunoassay, with an enzyme tracer, and when repeated measurements of the

293 same individuals were collected across a relatively short time span (i.e., a sampling interval of 8-

29414 days).

295 The repeatability of GCs within individuals can be used to: 1) determine whether

296 inferences made about GC measures may be generalized beyond the time of sampling (e.g.,

297 Bosson et al., 2009; Harris et al., 2016; Wada et al., 2008), 2) describe the ability or strategy of

298 an individual to cope with a challenge (Careau, Buttemer \& Buchanan, 2014; Roche, Careau \&

299 Binning, 2016), and 3) approximate the upper limit of heritability of individual variation and,

300 thereby, the extent to which natural selection can shape a trait (Falconer and Mackay 1996; but

301 see Dohm, 2002). Below, we interpret our findings in light of each of these applications of

302 estimates of repeatability. 
304 Angelier et al., 2010; Ferrari et al., 2013; and Narayan et al., 2013b) and, therefore, expected to

305 be reliable indicators of an individual's endocrine phenotype beyond the period of sampling,

306 many other measures were not. Low repeatability may be caused by high within-individual

307 variation, high measurement error, low among-individual variation, or a combination of all three.

308 Whether a population exhibits low repeatability due to high within-individual variation (rather

309 than low among-individual variation), or due to variation in trait consistency among individuals

310 has different implications for how to collect and interpret data from that population of

311 individuals (Jenkins, 2011; Biro \& Stamps, 2015). When high within-individual variation is a

312 concern, a single measurement of GCs will best capture individual differences when collected

313 from all individuals instantaneously or while controlling for as many sources of environmental

314 variation as possible. In the case of variation among individuals in trait consistency, a single

315 measure of GCs will be unlikely to capture how individuals differ overall.

316 Whether or not an endocrine trait is repeatable for a given population, if individuals are

317 sampled across different physical or social environments, or if some individuals differ in

318 personality-related strategies, then the within-individual relationship between hormones and

319 another variable of interest can differ from the population-level response in unexpected ways

320 (Roche et al., 2016). For example, while a study found no relationship between brood size and

321 baseline GCs among female tree swallows (Tachycineta bicolor), baseline GCs increased within

322 individuals following an experimental increase in brood size (Bonier, Moore \& Robertson,

323 2011). Additionally, olive flounder (Paralichthys olivaceus) with bold behavioral phenotypes

324 responded physiologically to an acute stress in a manner opposite that of shy types, and these

325 divergent responses were repeatable (Rupia et al., 2016). In both of these cases, failure to 
326 measure within-individual changes in GCs, or to recognize among-individual variation in the

327 direction of those responses, would have obscured detection of the effects of the challenge of

328 interest (i.e., brood size, acute stress) at the population level. Our finding of relatively low GC

329 repeatability, particularly for initial GCs, strongly suggests that these measures frequently reflect

330 an individual's short-term response to the environment more so than fixed differences among

331 individuals.

332 Variation in GC repeatability can also be used to investigate differences in the ability or

333 strategy of individuals or populations to respond to environmental change. For example, our

334 finding of significantly greater repeatability in response, compared to initial, measures could

335 indicate relatively greater canalization in the acute activation of the HPA axis, and a reduced

336 plasticity of this trait within individuals. Consistent with this interpretation, previous studies have

337 estimated greater realized heritability of the GC response in genetic lines selected for high, rather

338 than low, stress responses (Brown \& Nestor, 1973; Satterlee \& Johnson, 1988). Additionally, the

339 greater repeatability of both initial and response GCs in amphibians could indicate different

340 functions and/or responsiveness of the HPA axis in amphibians compared to other taxonomic

341 classes (Narayan et al., 2013a). Finally, our finding greater repeatability of response, but not

342 initial, GCs measured within a life history stage somewhat aligns with previous work, which has

343 shown greater seasonal variation in baseline, rather than stress-induced, GC titers (Romero,

344 2002). And although our sample size was small $(n=8)$, our finding of greater repeatability of

345 integrated $\mathrm{GC}$ measures during the non-breeding season seems to suggest less variation within

346 individuals in the total secretion of GCs during that period, which could reflect a broader pattern

347 of seasonal GC secretion across taxa. 
If one aims to compare repeatability or trait consistency among individuals, populations,

349 or even species, as described above, then an important consideration is whether variation among

350 repeatability estimates is due to laboratory or statistical methodologies impacting within- or

351 among-individual variation in the trait of interest. We found that some repeatability estimates

352 were lower when measured with a commercial kit compared to an in-house assay, and when

353 measured with an RIA as compared to an EIA. Commercial assay kits can be less precise (as

354 well as less accurate) in measuring GC concentrations if they are not carefully validated for the

355 study system (Buchanan \& Goldsmith, 2004; Sheriff et al., 2011), which may explain lower

356 repeatability estimates for GCs measured with kits. Further, the ease of use of commercial kits

357 might lend itself to less precise lab practices than the more involved in-house assays. However, it

358 is not clear why RIAs would be associated with lower repeatability. Brown et al. (2010) found

359 that, while urinary cortisol assessed with either RIA or EIA exhibited qualitatively-similar

360 temporal profiles, the RIA detected proportionally lower hormone concentrations (i.e., decreased

361 among-individual variation). This lower among-individual variation could lead to lower

362 repeatability, if it is not counteracted by simultaneously lower within-individual variation.

363 Previous work has documented large inter-laboratory variation in measurements of absolute

364 steroid hormone concentrations (Bókony et al., 2009; Ganswindt et al., 2012; Feswick et al.,

365 2014; Fanson et al., 2017), suggesting that across-study comparisons of absolute values of

366 individuals' GC titers are not valid. Finally, while we also found some evidence that response

367 GC repeatability was greater when repeated measurements were collected over a relatively short

368 time span (i.e., 8-14 days apart), even shorter time spans did not show a consistent pattern, and

369 we did not detect a similar effect in any of the other GC measures. Overall, if one seeks to

370 investigate the causes and consequences of variable GC repeatability among groups, to better 
371 understand the ability or strategy of these groups to respond to environmental conditions,

372 methodological sources of variation must be considered and, ideally, controlled.

373 A final application of estimates of trait repeatability is to approximate the upper limit of

374 heritability. The average repeatability of initial and response GCs reported here align well with

375 the results of artificial selection and animal model approaches that estimate a similar degree of

376 heritability in GC titers and the GC response (Evans et al., 2006; Jenkins et al., 2014; Pottinger

377 \& Carrick, 1999; Touma et al., 2008). These studies often find that the heritability of baseline

378 GCs is much lower than response GCs, if it is detectable at all (e.g., Brown \& Nestor, 1973;

379 Satterlee \& Johnson, 1988; Evans et al., 2006). Thus, we expect baseline concentrations will be

380 less likely to exhibit evolutionary change than stress-induced concentrations, when exposed to

381 similar selective pressures. Furthermore, Jenkins et al. (2014) failed to find phenotypic or genetic

382 correlations between baseline and stress-induced concentrations within individuals. This finding

383 suggests that different mechanisms may control GC secretion during normal activity versus

384 during challenging events, and that selection could affect variation in these traits independently

385 (Jenkins et al., 2014). As a result, selective or ecological pressures should be expected to produce

386 complex, context-dependent relationships between hormone titers and factors of interest.

387 Altogether, the low-to-moderate repeatability and heritability of GC titers underscores the extent

388 to which plasticity may generate individual variation, as well as the extent to which that variation 389 may be transmitted to future generations.

390 While our meta-analysis of GC repeatability estimates allowed us to look for patterns in

391 trait consistency across a range of methodological and biological factors, there are limitations to

392 our dataset and thus our ability to draw strong inferences from it. For example, many studies

393 calculated repeatability as a way to compliment or support their main results. If researchers are 
394 more likely to report repeatability estimates that support their main findings, then repeatability

395 estimates available in the literature may overestimate true repeatability. In addition, our

396 categorization of the biological and methodological data associated with each repeatability

397 estimate could have over-simplified or otherwise misrepresented the reality of the study, which

398 could make real patterns more difficult to detect, or possibly cause spurious patterns (e.g., among

399 the more weakly-supported findings). Finally, sample size was limited for many categories

400 included in our analyses, thereby reducing our statistical power to detect real patterns.

401

402 Conclusion

403 Overall, this meta-analysis provides new insights into individual variation in GC titers,

404 and highlights the importance of repeatability estimation to improve methods for collecting and

405 interpreting biological data. We found that GCs were moderately repeatable, on average, but

406 these estimates were also highly variable. Additionally, initial and response GC measures were

407 more repeatable in amphibians than any other taxonomic class, while response GCs were more

408 repeatable when measured within the same life history stage and integrated GC were more

409 repeatable during the non-breeding season. We look forward to new research that further

410 investigates how and why repeatability differs with these factors. However, our finding that

411 laboratory techniques were also associated with variation in repeatability could serve as a

412 reminder to be meticulous in monitoring for issues with the reproducibility of hormone data.

413 Moving forward, a better understanding of endocrine trait evolution requires knowledge about

414 heritable individual differences in evolutionarily-important traits. Our analysis shows that a

415 single measure of individual variation in GC titers may not reflect how those individuals differ in 
416 general, and suggests different approaches to capture that signal, including repeated

417 measurements of individuals both within and across environments. 
419 Acknowledgments

420 We thank Y. Aharon-Rotman, A. Gladbach, B. Dantzer, J. Riechert, C. Vleck, H. Wada, and S.

421 Winberg for providing data or additional information about their published studies included in

422 the analyses. We also thank R. Montgomerie for advice on the statistical analyses. We are

423 grateful for the constructive remarks of N. Cyr and two anonymous referees, which improved the 424 final manuscript. 
425 References

426 Angelier F., Wingfield JC., Weimerskirch H., Chastel O. 2010. Hormonal correlates of

427

428

429

430

431

432

433

434

435

436

437

438

439

440

441

442

443

444

445

446

447

448

449

450

451

452

453

454

455

456

457

458

459

460

461

462

463

464

465

466

467

468

469

470 individual quality in a long-lived bird: a test of the "corticosterone-fitness hypothesis". Biology letters 6:846-849. DOI: 10.1098/rsbl.2010.0376.

Bell AM., Hankison SJ., Laskowski KL. 2009. The repeatability of behaviour: a meta-analysis. Animal Behaviour 77:771-783. DOI: 10.1016/j.anbehav.2008.12.022.

Bennett AF. 1987. Inter-individual variability: an under-utilized resource. In: Feder ME, Bennett AF, Burggren WW \& Huey RB, eds. New directions in ecological physiology. Cambridge University Press: Cambridge, 147-169.

Biro PA., Stamps JA. 2015. Using repeatability to study physiological and behavioural traits: ignore time-related change at your peril. Animal Behaviour 105:223-230. DOI: 10.1016/j.anbehav.2015.04.008.

Bizon JL., Helm KA., Han J., Chun H., Pucilowska J., Lund PK., Gallagher M. 2001. Hypothalamic-pituitary-adrenal axis function and corticosterone receptor expression in behaviourally characterized young and aged Long-Evans rats. Neuroscience 14:1739-1751.

Bókony V., Lendvai ÁZ., Liker A., Angelier F., Wingfield JC., Chastel O. 2009. Stress response and the value of reproduction: are birds prudent parents? The American naturalist 173:589598. DOI: $10.1086 / 597610$.

Bonier F., Martin PR. 2016. How can we estimate natural selection on endocrine traits? Lessons from evolutionary biology. Proceedings of the Royal Society B 283:20161887. DOI: 10.1098/rspb.2016.1887.

Bonier F., Martin PR., Moore IT., Wingfield JC. 2009. Do baseline glucocorticoids predict fitness? Trends in Ecology and Evolution 24:634-642. DOI: 10.1016/j.tree.2009.04.013.

Bonier F., Moore IT., Robertson RJ. 2011. The stress of parenthood? Increased glucocorticoids in birds with experimentally enlarged broods. Biology Letters 7:944-946. DOI: 10.1098/rsbl.2011.0391.

Bosson CO., Palme R., Boonstra R. 2009. Assessment of the stress response in Columbian ground squirrels: laboratory and field validation of an enzyme immunoassay for fecal cortisol metabolites. Physiological and biochemical zoology 82:291-301. DOI: $10.1086 / 597530$.

Boswell T., Woods SC., Kenagy GJ. 1994. Seasonal changes in body mass, insulin, and glucocorticoids of free-living golden-mantled ground squirrels. General and comparative endocrinology 96:339-346. DOI: 10.1006/gcen.1994.1189.

Breuner CW., Lynn SE., Julian GE., Cornelius JM., Heidinger BJ., Love OP., Sprague RS., Wada H., Whitman BA. 2006. Plasma-binding globulins and acute stress response. Hormone and Metabolic Research 38:260-268. DOI: 10.1055/s-2006-925347.

Brown JL., Kersey DC., Freeman EW., Wagener T. 2010. Assessment of diurnal urinary cortisol excretion in Asian and African elephants using different endocrine methods. Zoo Biology 29:274-283. DOI: 10.1002/zoo.20268.

Brown KI., Nestor KE. 1973. Some physiological responses of turkeys selected for high and low adrenal response to cold stress . Poultry science 52:1948.

Buchanan KL., Goldsmith AR. 2004. Noninvasive endocrine data for behavioural studies: The importance of validation. Animal Behaviour 67:183-185. DOI:

10.1016/j.anbehav.2003.09.002.

Careau V., Buttemer WA., Buchanan KL. 2014. Early-developmental stress, repeatability, and canalization in a suite of physiological and behavioral traits in female zebra finches. 
484

485

486

487

488

489

490

491

492

493

494

495

496

497

498

499

500

501

502

503

504

505

506

507

508

509

510

511

512

513

514

515

516

Integrative and comparative biology 54:539-554. DOI: 10.1093/icb/icu095.

Cook K V., O’Connor CM., McConnachie SH., Gilmour KM., Cooke SJ. 2012. Condition dependent intra-individual repeatability of stress-induced cortisol in a freshwater fish. Comparative Biochemistry and Physiology, Part A 161:337-343. DOI:

10.1016/j.cbpa.2011.12.002.

Dohm MR. 2002. Repeatability estimates do not always set an upper limit to heritability. Functional Ecology 16:273-280. DOI: 10.1046/j.1365-2435.2002.00621.x.

Egger M., Smith GD., Schneider M., Minder C. 1997. Bias in meta-analysis detected by a simple , graphical test measures of funnel plot asymmetry. Bmj 315:629-34. DOI:

10.1136/bmj.315.7109.629.

Eikenaar C., Klinner T., Stöwe M. 2014. Corticosterone predicts nocturnal restlessness in a longdistance migrant. Hormones and Behavior 66:324-329. DOI: 10.1016/j.yhbeh.2014.06.013.

Ekins RP. 1960. The estimation of thyroxine in human plasma by an electrophoretic technique. Clinica chimica acta; international journal of clinical chemistry 5:453-459. DOI: 10.1016/0009-8981(60)90051-6.

Evans MR., Roberts ML., Buchanan KL., Goldsmith AR. 2006. Heritability of corticosterone response and changes in life history traits during selection in the zebra finch. Journal of Evolutionary Biology 19:343-352. DOI: 10.1111/j.1420-9101.2005.01034.x.

Falconer DS., Mackay TF. 1996. Introduction to Quantitative Genetics, $4^{\text {th }}$ edn. Longman, Harlow.

Fanson K V., Németh Z., Ramenofsky M., Wingfield JC., Buchanan KL. 2017. Inter-laboratory variation in corticosterone measurement: Implications for comparative ecological and evolutionary studies. Methods in Ecology and Evolution:1-10. DOI: 10.1111/2041210X.12821.

Ferrari C., Pasquaretta C., Carere C., Cavallone E., von Hardenberg A., Réale D. 2013. Testing for the presence of coping styles in a wild mammal. Animal Behaviour 85:1385-1396. DOI: 10.1016/j.anbehav.2013.03.030.

Feswick A., Ankley GT., Denslow N., Ellestad LE., Fuzzen M., Jensen KM., Kroll K., Lister A., Maclatchy DL., Mcmaster ME., Orlando EF., Servos MR., Tetreault GR., Van Den Heuvel MR., Munkittrick KR. 2014. An inter-laboratory study on the variability in measured concentrations of 17 $\beta$-estradiol, testosterone, and 11-ketotestosterone in white sucker: implications and recommendations. Environmental Toxicology and Chemistry 33:847-857. DOI: $10.1002 /$ etc. 2503.

Ganswindt A., Brown JL., Freeman EW., Kouba AJ., Penfold LM., Santymire RM., Vick MM., Wielebnowski N., Willis EL., Milnes MR. 2012. International Society for Wildlife Endocrinology: the future of endocrine measures for reproductive science, animal welfare and conservation biology. Biology Letters Physiology 8:695-697. DOI: 10.1098/rsbl.2011.1181.

Halekoh U., Højsgaard S. 2014. A Kenward-Roger Approximation and Parametric Bootstrap Methods for Tests in Linear Mixed Models - The R Package pbkrtest. Journal of Statistical Software 59:1-32. DOI: 10.18637/jss.v059.i09.

Harris CM., Madliger CL., Love OP. 2016. Temporal overlap and repeatability of feather corticosterone levels: practical considerations for use as a biomarker. Conservation Physiology 4:1-11. DOI: 10.1093/conphys/cow051.

Heidinger BJ., Nisbet ICT., Ketterson ED. 2006. Older parents are less responsive to a stressor in a long-lived seabird: a mechanism for increased reproductive performance with age? 
517

518

519

520

521

522

523

524

525

526

527

528

529

530

531

532

533

534

535

536

537

538

539

540

541

542

543

544

545

546

547

548

549

550

551

552

553

554

555

556

557

558

559

560

561

562

Proceedings of the Royal Society B: Biological Sciences 273:2227-2231. DOI: 10.1098/rspb.2006.3557.

Jenkins SH. 2011. Sex differences in repeatability of food-hoarding behaviour of kangaroo rats. Animal Behaviour 81:1155-1162. DOI: 10.1016/j.anbehav.2011.02.021.

Jenkins BR., Vitousek MN., Hubbard JK., Safran RJ. 2014. An experimental analysis of the heritability of variation in glucocorticoid concentrations in a wild avian population. Proceedings of the Royal Society B 281:20141302. DOI: 10.1098/rspb.2014.1302.

Kenward MG., Roger JH. 1997. Small Sample Inference for Fixed Effects from Restricted Maximum Likelihood. Biometrics 53:983. DOI: 10.2307/2533558.

Klosterman LL., Murai JT., Siiteri PK. 1986. Cortisol levels, binding, and properties of corticosteroid-binding globulin in the serum of primates. Endocrinology 118:424-434. DOI: 10.1210/endo-118-1-424.

Kuznetsova A., Brockhoff PB., Christensen RHB. 2016. lmerTest: Tests in Linear Mixed Effects Models, R package version 2.0-33. https://CRAN.R-project.org/package=lmerTest

Lattin CR., Romero LM. 2014. Chronic stress alters concentrations of corticosterone receptors in a tissue-specific manner in wild house sparrows (Passer domesticus). Journal of Experimental Biology 217:2601-2608. DOI: 10.1242/jeb.103788.

Liebl AL., Shimizu T., Martin LB. 2013. Covariation among glucocorticoid regulatory elements varies seasonally in house sparrows. General and Comparative Endocrinology 183:32-37. DOI: 10.1016/j.ygcen.2012.11.021.

McEwen BS., Wingfield JC. 2007. Allostasis and allostatic load. In: Fink G, ed. Encyclopedia of Stress 2. New York: Academic Press, 135-141.

Nakagawa S., Santos ESA. 2012. Methodological issues and advances in biological metaanalysis. Evolutionary Ecology 26:1253-1274. DOI: 10.1007/s10682-012-9555-5.

Nakagawa S., Schielzeth H. 2010. Repeatability for Gaussian and non-Gaussian data: a practical guide for biologists. Biological Reviews 85:935-956. DOI: 10.1111/j.1469185X.2010.00141.x.

Narayan EJ., Cockrem JF., Hero JM. 2013a. Are baseline and short-term corticosterone stress responses in free-living amphibians repeatable? Comparative Biochemistry and Physiology, Part A 164:21-28. DOI: 10.1016/j.cbpa.2012.10.001.

Narayan EJ., Cockrem JF., Hero JM. 2013b. Repeatability of baseline corticosterone and shortterm corticosterone stress responses, and their correlation with testosterone and body condition in a terrestrial breeding anuran (Platymantis vitiana). Comparative Biochemistry and Physiology - A Molecular and Integrative Physiology 165:304-312. DOI: 10.1016/j.cbpa.2013.03.033.

Nespolo RF., Franco M. 2007. Whole-animal metabolic rate is a repeatable trait: a meta-analysis. The Journal of Experimental Biology 210:2000-5. DOI: 10.1242/jeb.02780.

Ouyang JQ., Hau M., Bonier F. 2011. Within seasons and among years: When are corticosterone levels repeatable? Hormones and Behavior 60:559-564. DOI: 10.1016/j.yhbeh.2011.08.004.

Pancak MK., Taylor H. 1983. Seasonal and daily plasma corticosterone rhythms in American toads, Bufo americanus. General and Comparative Endocrinology 50:490-497. DOI: 0016480/83.

Pottinger TG., Carrick TR. 1999. Modification of the Plasma Cortisol Response to Stress in Rainbow Trout by Selective Breeding. General and Comparative Endocrinology 116:122132. DOI: http://dx.doi.org/10.1006/gcen.1999.7355. 
563 Roche DG., Careau V., Binning SA. 2016. Demystifying animal "personality" (or not): why 564 individual variation matters to experimental biologists. Journal of Experimental Biology. 565 DOI: $10.1242 /$ jeb. 146712.

566

567

568

569

570

571

572

573

574

575

576

577

578

579

580

581

582

583

584

585

586

587

588

589

590

591

592

593

594

595

596

597

598

600

601

602

603

604

605

606

Romero LM. 2004. Physiological stress in ecology: lessons from biomedical research. Trends in Ecology and Evolution 19:249-255. DOI: 10.1016/j.tree.2004.03.008.

Romero LM., Dickens MJ., Cyr NE. 2009. The reactive scope model: a new model integrating homeostasis, allostasis, and stress. Hormones and Behavior 55:375-389. DOI: 10.1016/j.yhbeh.2008.12.009.

Romero LM., Reed JM. 2008. Repeatability of baseline corticosterone concentrations. General and Comparative Endocrinology 156:27-33. DOI: 10.1016/j.ygcen.2007.10.001.

Rupia EJ., Binning SA., Roche DG., Lu W. 2016. Fight-flight or freeze-hide? Personality and metabolic phenotype mediate physiological defence responses in flatfish. Journal of Animal Ecology 85:927-937. DOI: 10.1111/1365-2656.12524.

Sapolsky RM., Romero LM., Munck AU. 2000. How do glucocorticoids influence stress responses? Integrating suppressive, stimulatory, and preparative actions. Endocrine Reviews 21:55-89. DOI: 10.1210/er.21.1.55.

Satterlee DG., Johnson WA. 1988. Selection of Japanese quail for contrasting blood corticosterone response to immobilization. Poultry Science 67:25-32. DOI: $10.3382 /$ ps.0670025.

Sheriff MJ., Dantzer B., Delehanty B., Palme R., Boonstra R. 2011. Measuring stress in wildlife: Techniques for quantifying glucocorticoids. Oecologia 166:869-887. DOI: 10.1007/s00442-011-1943-y.

Sokal RR., Rohlf FJ. 1995. Biometry: The principles and practice of statistics in biological research, $3^{\text {rd }}$ edn. W.H. Freemand and Company, New York.

Stinchcombe JR., Rutter MT., Burdick DS., Tiffin P., Rausher MD., Mauricio R. 2002. Testing for Environmentally Induced Bias in Phenotypic Estimates of Natural Selection: Theory and Practice. The American Naturalist 160:511-523. DOI: 10.1086/342069.

Touma C., Bunck M., Glasl L., Nussbaumer M., Palme R., Stein H., Wolferstätter M., Zeh R., Zimbelmann M., Holsboer F., Landgraf R. 2008. Mice selected for high versus low stress reactivity: a new animal model for affective disorders. Psychoneuroendocrinology 33:839862. DOI: $10.1016 /$ j.psyneuen.2008.03.013.

Wada H., Salvante KG., Stables C., Wagner E., Williams TD., Breuner CW. 2008. Adrenocortical responses in zebra finches (Taeniopygia guttata): individual variation, repeatability, and relationship to phenotypic quality. Hormones and Behavior 53:472-480. DOI: 10.1016/j.yhbeh.2007.11.018.

White CR., Schimpf NG., Cassey P. 2013. The repeatability of metabolic rate declines with time. Journal of Experimental Biology 216:1763-1765. DOI: 10.1242/jeb.076562.

Williams TD. 2008. Individual variation in endocrine systems: moving beyond the "tyranny of the Golden Mean." Philosophical Transactions of the Royal Society B: Biological Sciences 363:1687-1698. DOI: 10.1098/rstb.2007.0003.

Wingfield JC. 2005. The concept of allostasis: coping with a capricious environment. Journal of Mammalogy 86:248-254. DOI: 10.1644/BHE-004.1.

Yalow RS., Berson SA. 1960. Immunoassay of endogneous plasma insulin in man. Journal of Clinical Investigation 39:1157-1175. DOI: 10.1172/JCI104130. 


\section{Table $\mathbf{1}$ (on next page)}

List describing how methodological and biological factors associated with each repeatability estimate were categorized for analysis. 
1 Table 1. List describing how methodological and biological factors associated with each

2 repeatability estimate were categorized for analysis.

3

\section{FACTOR CATEGORIES}

Time between measurements ${ }^{1}$

0-7d, 8-14d, 15-30d, 31-90d, 91-195d, or 365+

Number of

measurements ${ }^{1}$

Two, more than 2

Captive

condition

Taxonomic

class

Age

$\operatorname{Sex}$

\section{Life history stage (LHS)}

\section{Measured} within LHS

Assay source

Assay tracer

Experimental manipulation $^{3}$

Adjusted $^{4}$
Free-ranging, captive, wild-caught captive

Bird, mammal, amphibian, bony fish, reptile

Adult, juvenile, both

Male, female, both

Breeding, non-breeding, pre-breeding, $\mathrm{NA}^{2}$

Yes, No

In-house, commercial kit

Radioactive, enzymatic

Yes, No

Yes, No

${ }^{1}$ Average, weighted by number of individuals when possible

$5{ }^{2}$ We categorized life history stage as "NA" for domesticated or captive-born species because

6 domestication can alter seasonal patterns in hormone physiology (Donham, 1979; Sossinka, 1982; Künzl

$7 \&$ Sachser, 1999). Estimates from these species were not included in analyses that examined the effect of

8 life history stage.

$9{ }^{3}$ Experimental manipulation refers to studies in which some or all individuals underwent a stressful

10 manipulation intended to produce a response (not including routine capture and handling stress) at some

11 point during the course of the study.

$12{ }^{4}$ Adjusted refers to whether or not estimates reflect GC repeatability after statistically controlling for

13 factors expected to explain some of the variation in GC titers (e.g., year, sex, weather). 


\section{Table 2 (on next page)}

Summary of the data included in the meta-analysis.

Except for sample size, numbers provided reflect the number of estimates in each category. 
1 Table 2. Summary of the data included in the meta-analysis. Except for sample size, numbers

2 provided reflect the number of estimates in each category.

\begin{tabular}{|c|c|c|c|c|c|c|}
\hline GC measure & $\begin{array}{c}\text { Initial }^{1} \\
42\end{array}$ & $\begin{array}{c}\text { Response }^{2} \\
37\end{array}$ & $\begin{array}{c}\text { Integrated }^{\mathbf{3}} \\
12\end{array}$ & & & \\
\hline Sample size & $\begin{array}{c}\text { Mean } \\
36 \pm \text { SE } 4.5\end{array}$ & $\begin{array}{l}\text { Range } \\
8-352\end{array}$ & & & & \\
\hline $\begin{array}{c}\text { Sampling } \\
\text { interval }\end{array}$ & $\begin{array}{c}\mathbf{0 - 7 d} \\
13\end{array}$ & $\begin{array}{c}\mathbf{8 - 1 4 d} \\
26\end{array}$ & $\begin{array}{c}\text { 15-30d } \\
8\end{array}$ & $\begin{array}{c}\text { 31-90d } \\
17\end{array}$ & $\begin{array}{c}\text { 91-195d } \\
4\end{array}$ & $\begin{array}{c}365+d \\
23\end{array}$ \\
\hline $\begin{array}{c}\text { Number of } \\
\text { measurements }\end{array}$ & $\begin{array}{c}2 \\
39\end{array}$ & $\begin{array}{l}>2 \\
52\end{array}$ & & & & \\
\hline $\begin{array}{c}\text { Captive } \\
\text { condition }\end{array}$ & $\begin{array}{c}\text { Free-ranging } \\
58\end{array}$ & $\begin{array}{c}\text { Captive-born } \\
14\end{array}$ & $\begin{array}{c}\text { Wild-caught } \\
\text { captive } \\
19\end{array}$ & & & \\
\hline Taxonomic class & $\begin{array}{c}\text { Bird } \\
60\end{array}$ & $\begin{array}{c}\text { Mammal } \\
11\end{array}$ & $\begin{array}{c}\text { Amphibian } \\
8\end{array}$ & $\begin{array}{c}\text { Bony fish } \\
9\end{array}$ & $\begin{array}{c}\text { Reptile } \\
3\end{array}$ & \\
\hline Age & $\begin{array}{c}\text { Adult } \\
80\end{array}$ & $\begin{array}{c}\text { Juvenile } \\
2\end{array}$ & $\begin{array}{c}\text { Both } \\
9\end{array}$ & & & \\
\hline Sex & $\begin{array}{c}\text { Male } \\
18\end{array}$ & $\begin{array}{c}\text { Female } \\
30\end{array}$ & $\begin{array}{c}\text { Both } \\
43\end{array}$ & & & \\
\hline $\begin{array}{c}\text { Life history } \\
\text { stage (LHS) }\end{array}$ & $\begin{array}{c}\text { Breeding } \\
36\end{array}$ & $\begin{array}{c}\text { Non-breeding } \\
21\end{array}$ & $\begin{array}{c}\text { Pre-breeding } \\
9\end{array}$ & $\begin{array}{c}\mathbf{N A} \\
25\end{array}$ & & \\
\hline Within LHS & $\begin{array}{c}Y \\
64\end{array}$ & $\begin{array}{l}\mathbf{N} \\
11\end{array}$ & $\begin{array}{c}\mathbf{N A}^{4} \\
16\end{array}$ & & & \\
\hline Assay source & $\begin{array}{c}\text { In-house } \\
51\end{array}$ & $\begin{array}{c}\text { Kit-based } \\
35\end{array}$ & & & & \\
\hline Assay tracer & $\begin{array}{c}\text { Radioactive } \\
42\end{array}$ & $\begin{array}{c}\text { Enzyme } \\
44\end{array}$ & & & & \\
\hline $\begin{array}{l}\text { Experimental } \\
\text { manipulation }^{5}\end{array}$ & $\begin{array}{c}\mathbf{Y} \\
21\end{array}$ & $\begin{array}{c}\mathbf{N} \\
70\end{array}$ & & & & \\
\hline Adjusted $^{6}$ & $\begin{array}{c}\mathbf{Y} \\
21\end{array}$ & $\begin{array}{c}\mathbf{N} \\
70\end{array}$ & & & & \\
\hline \multicolumn{7}{|c|}{$\begin{array}{l}{ }^{1} \text { Initial GCs refer to concentrations of GCs expected not to reflect the acute stress of capture. } \\
{ }^{2} \text { Response GCs refer to elevated GC titers following an acute capture, handling, or confinement stress. } \\
{ }^{3} \text { Integrated GCs refer to GC titers representing hormone secretion over a relatively long time. } \\
{ }^{4} \text { We categorized life history stage as "NA" for domesticated or captive-born species because } \\
\text { domestication can alter seasonal patterns in hormone physiology. Estimates from these species were not } \\
\text { included in analyses that examined the effect of life history stage. } \\
{ }^{5} \text { Experimental manipulation refers to studies in which some or all individuals underwent a stressful } \\
\text { manipulation intended to produce a response (not including routine capture and handling stress) at some } \\
\text { point during the course of the study. } \\
{ }^{6} \text { Adjusted refers to whether or not estimates reflect GC repeatability after statistically controlling for } \\
\text { factors expected to explain some of the variation in GC titers (e.g., year, sex, weather). }\end{array}$} \\
\hline
\end{tabular}




\section{Figure 1}

\section{PRISMA flow diagram}

Preferred Reporting Items for Systematic Reviews and Meta-analysis (PRISMA) flowchart illustrating the process of study identification, screening, and inclusion in the meta-analysis.

Footnotes:

${ }^{1}$ We used the search terms: repeatab*, consisten*, glucocorticoid, cortisol, corticoster*, repeated measure, individual variation

${ }^{2}$ We included three studies that did not meet inclusion criteria (i.e., collected repeated within individuals, but did not estimate repeatability) because we were able obtain the original data from the study authors and calculate repeatability ourselves.

${ }^{3}$ We used the following inclusion criteria: the study had to assess repeated measurements of glucocorticoids within the same individual, and estimate a repeatability coefficient (e.g., Spearman rank, Pearson, or intraclass correlation coefficient). 


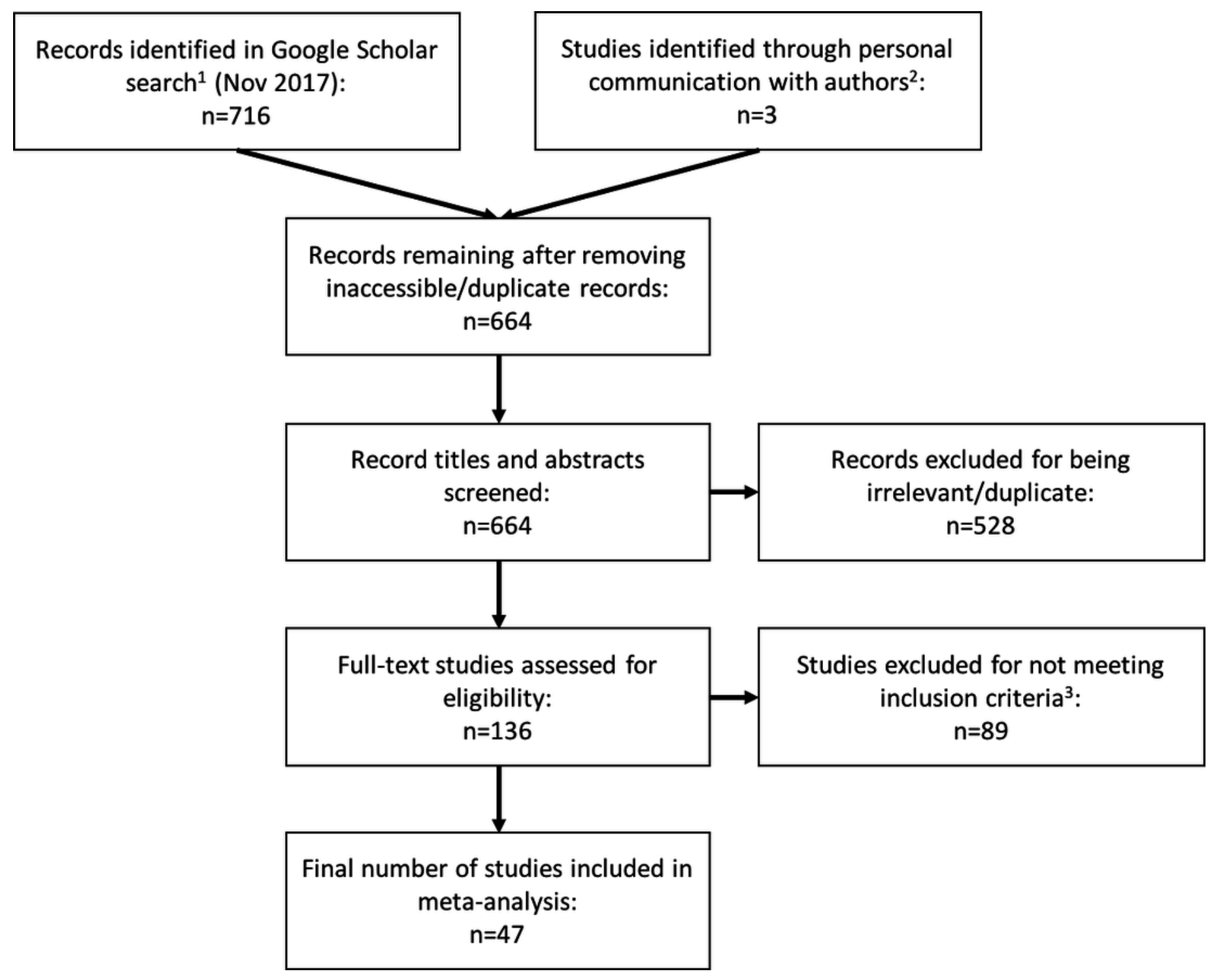




\section{Figure 2}

Frequency distributions of all estimates of repeatabilities of A) initial, B) response, and C) integrated glucocorticoid (GC) measures included in the meta-analyses.

The mean repeatability across all estimates of each category of GC is represented by a solid line, and the $95 \% \mathrm{Cl}$ (calculated from 1000 bootstrap samples of the data with replacement) is represented by a dashed line. In this study, we defined initial measures as those representing GCs in circulation within a time period expected not to reflect the acute stress of capture, response for elevated GC titers following an acute capture stress, and integrated for GC titers that represent hormone secretion over a relatively long period of time (e.g., GC concentrations in feces, feathers, and saliva). 


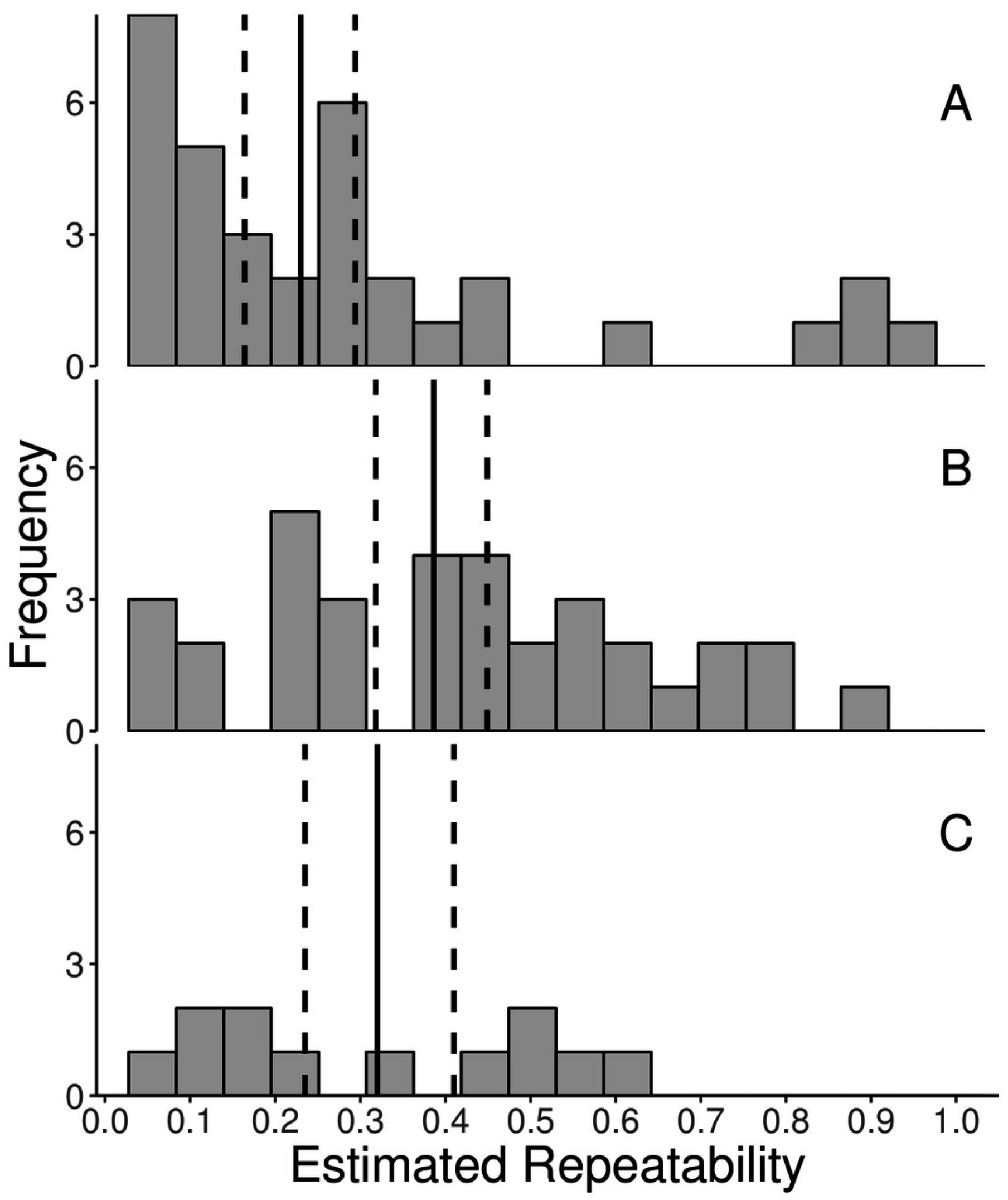




\section{Figure 3}

Boxplots showing variation in the average repeatability of all glucocorticoid (GC) measures across taxonomic classes (data are jittered along $\mathrm{x}$-axis for ease of interpretation).

The plot's whiskers represent the 1.5 interquartile range, while the boxes represent the first and third quartiles, and the midline represents the median. Repeatability estimates for initial (open circles) and response (open triangles), but not integrated (closed squares), GC measures varied across taxonomic class (Type III ANOVA; initial: $n=38, F(3,38)=9.359$, $p<0.0001$; response: $n=27, F(4,23)=4.984, p=0.005)$. In this study, we defined initial measures as those representing GCs in circulation within a time period expected not to reflect the acute stress of capture, response for elevated GC titers following an acute capture stress, and integrated for GC titers that represent hormone secretion over a relatively long period of time (e.g., GC concentrations in feces, feathers, and saliva). 


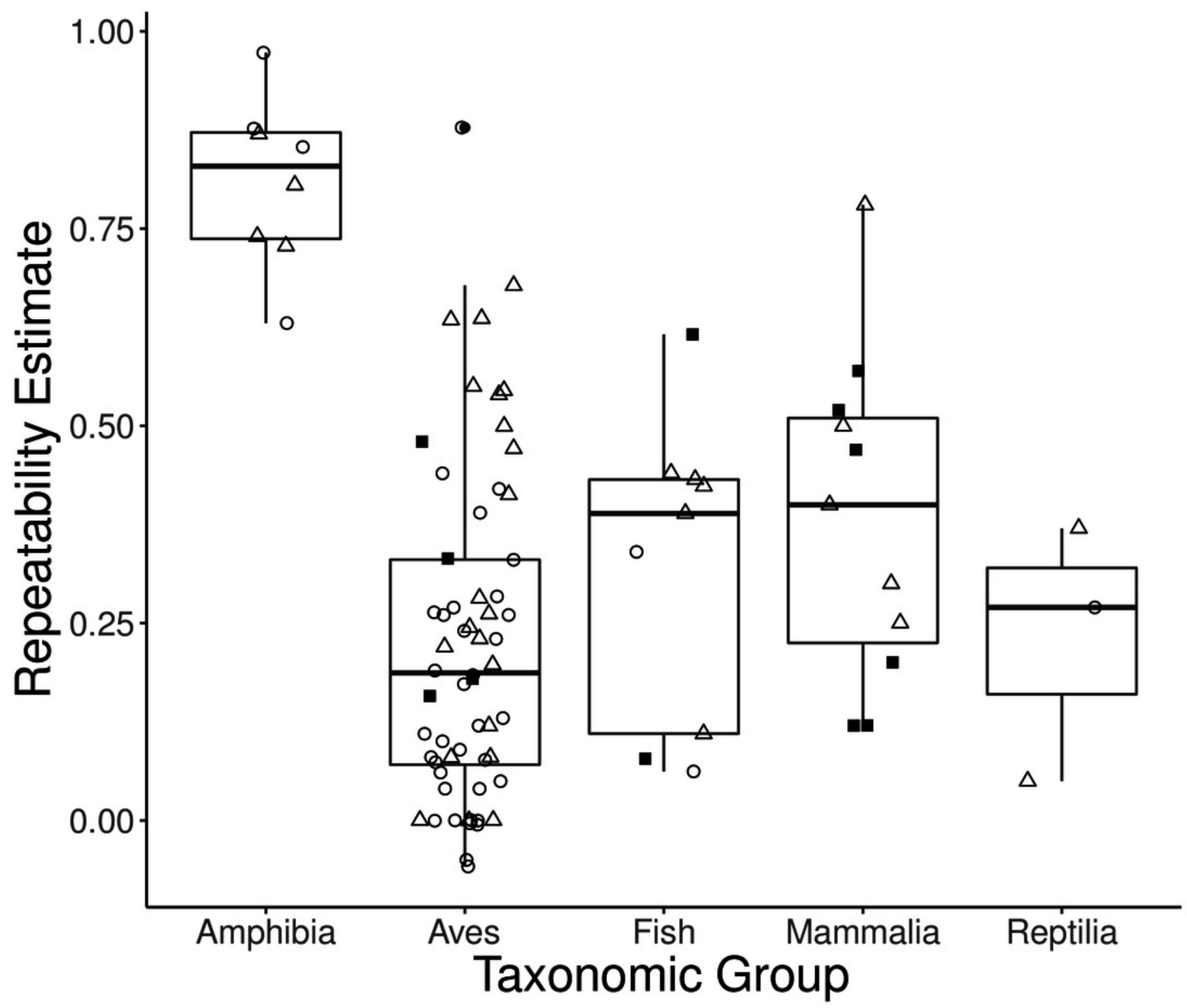

